This is the peer reviewed version of the following article: Escalada-Hermández, P. and Marín-Fernández, B. (2018), The Nursing Diagnosis Disturbed Thought Processes: An Integrative Review. Int J Nurs Terminol Knowledge, 29: 253-262. doi:10.1111/2047-3095.12186, which has been published in final form at

\title{
The nursing diagnosis Disturbed Thought Processes: An integrative review
}

\author{
Paula Escalada-Hernández \\ $\mathrm{RN}, \mathrm{MsC}, \mathrm{PhD}$ \\ Assistant Professor, Department of Health Sciences, Public University of Navarre, Navarre, \\ Spain \\ E-mail: paula.escalada@unavarra.es \\ Blanca Marín-Fernández \\ RN, BSc Anthropology, PhD \\ Associate professor, Department of Health Sciences, Public University of Navarre, Navarre, \\ Spain \\ E-mail: $\underline{\text { blanca.marin@unavarra.es }}$
}

\section{CORRESPONDENCE TO:}

Paula Escalada Hernández

Department of Health Sciences

Public University of Navarre

Avenida de Barañain s/n. 31008, Pamplona, Navarra (SPAIN).

paula.escalada@unavarra.es

\begin{abstract}
:
PURPOSE: To analyze and synthetize the existing scientific literature in relation to the nursing diagnosis disturbed thought processes (DTPs) (00130).
\end{abstract}

METHODS: An integrative review was developed, identifying relevant papers through a search of international and Spanish databases and the examination of key manuals.

FINDINGS: Theoretical papers propose modifications for the nursing diagnosis DTPs. Most of the research papers offer data about its frequency in different clinical settings.

CONCLUSIONS: There exists an interest in the nursing diagnosis DTPs. However, the available evidence is not very extensive and further work is necessary in order to refine this nursing diagnosis.

IMPLICATIOS FOR NURSING PRACTICE: The reinclusion of DTPs in the NANDA-I classification will specially contribute to increment its utility in mental health care. 


\section{RESUMEN:}

OBJETIVO: Analizar y sintetizar la literatura científica existente en relación al diagnóstico enfermero trastorno de los procesos de pensamiento (DTPs) (00130).

MÉTODOS: Se desarrolló una revisión bibliográfica integradora, identificando los textos relevantes mediante una búsqueda en bases de datos internacionales y españolas y la revisión de manuales clave.

RESULTADOS: Los textos teóricos proponen modificaciones para el diagnóstico enfermero DTPs. La mayoría de los artículos de investigación ofrecen datos sobre su frecuencia en diferentes contextos clínicos.

CONCLUSIONES: Existe un interés en el diagnóstico de enfermería DTPs. Sin embargo, la evidencia disponible no es muy extensa y es preciso seguir trabajando para refinar este diagnóstico enfermero.

IMPLICACIONES PARA LA PRÁCTICA ENFERMERA: La reinclusión de DTPs en la clasificación NANDA-I contribuirá especialmente a incrementar su utilidad en salud mental.

\section{Problem Identification}

Currently, the NANDA-I taxonomy includes 235 different nursing diagnoses (Hederman \& Kamitsuru, 2014). Over the last decades, research focused on this classification has experienced a great impulse at an international level. This is contributing to improve the quality and rigor of the evidence that supports the existing nursing diagnoses. However, research also suggests that in certain clinical specialities, such as psychiatry, nurses, in their daily practice, address numerous patients' health problems that are not included in the NANDA-I classification (Frauenfelder, Müller-Staub, Needham, \& Van Achterberg, 2011; Frauenfelder, Van Achterberg, Needham, \& Müller Staub, 2016). This circumstance highlights the need for further work on the review of existing diagnoses and on the development of new ones in order to improve and complete this taxonomy (Echevarría Pérez, Romero Sánchez, Giró Formatger, \& Giménez Fernández, 2016; Hederman \& Kamitsuru, 2014).

The nursing diagnosis disturbed thought processes (00130) (DTPs), previously known as "altered thought processes", was included in the NANDA-I taxonomy since its first 
edition in 1973 (Gordon \& Sweeney, 1979) and later revised in 1996. This nursing diagnosis was defined as "disruption in cognitive operations and activities" (NANDA International, 2008, p. 408). Due to the lack of existing evidence in relation to its conceptual development and validation, this diagnosis was excluded from the classification in the 2012-2014 edition (Herdman, 2012). However, the nursing diagnosis DTPs is of special clinical relevance in the context of mental health care, since it has been proved to be one of the most frequently used nursing diagnosis within this field (Escalada Hernández, Muñoz Hermoso, \& Marro Larrañaga, 2013).

The exclusion of this nursing diagnosis from the NANDA-I taxonomy makes difficult its use in care plans and, as a consequence, nurses who address this human response in their daily practice are not able to document rigorously the care offered to their patients. Therefore, the implementation of research projects in order to increase the conceptual and empirical foundation of the nursing diagnosis DTPs and to support its reinclusion in the NANDA-I taxonomy is essential.

With the purpose of expanding the evidence base for the nursing diagnosis DTPs, a research project with two phases was undertaken. In the first phase, an exhaustive theoretical and conceptual review inspired the development of a new version of this diagnosis. In the second phase, this version was subjected to a content validation by a panel of experts. In this paper, the first step of the first phase of this research is presented: an integrative review aimed at analyzing and synthetizing the existing empirical evidence and scientific literature in relation to the nursing diagnosis DTPs.

\section{Methods}

The method of integrative review was chosen because it allows for gathering and synthesizing the existing evidence about a phenomenon of concern, combining data from both the empirical and the theoretical literature (Whittemore \& Knafl, 2005). In addition, the 
integrative review has been advocated as an initial step in the process of refinement and validation of nursing diagnoses (Pompeo, Rossi, \& Galvão, 2009).

\section{Search Methods}

Sources of literature. Multiple electronic databases were accessed, both international and Spanish ones (PubMed, Web of Science, CINAHL Complete, PsycInfo, Scopus, CUIDEN, ENFISPO, Hemeroteca Cantárida, IBECS and LILACS). Moreover, a manual search through the most relevant nursing journals was performed. Additional papers were selected from the references in the papers identified. Finally, in order to find theoretical contributions about the nursing diagnosis DTPs, manuals about nursing diagnoses considered as key were reviewed (Ackley \& Ladwig, 2007; Campbell, 1994; Carpenito, 2003; Doenges \& Moorhouse, 1992; Kim, McFarland, \& McLane, 1994; Luis, 1998; Luis Rodrigo, 2008; Ugalde Apalategui \& Rigol i Cuadra, 1995; Wilkinson \& Ahern, 2008).

Search terms. The expression "thought processes" is widely used in the fields of psychology and psychiatry. Thus, in order to design a search strategy as specific as possible, limited to the field of nursing, the search terms applied were the diagnostic label and the previous denomination of the diagnosis, both in quotation marks (i.e. "disturbed thought processes" OR "altered thought processes"). These search terms were introduced in either English or Spanish, according to the database.

Inclusion and exclusion criteria. As there were no previous literature reviews focused on this diagnosis, no publication date limit was applied. The papers selected were all those that provided relevant data about the nursing diagnosis DTPs and were written in either English, Spanish or Portuguese. Those theoretical reports that offered an analysis or review of the diagnosis and those empirical reports that presented empirical data about it were included. Reports of clinical cases and care plans were excluded. 
Search and selection process. The search was conducted in January 2017. From the search, 198 references were identified. After reviewing their titles and abstracts, 36 papers that complied with the selection criteria were finally selected.

\section{Data synthesis and quality appraisal}

The papers included in the review were classified into two categories:

theoretical reports and empirical reports. A formal assessment of the methodological quality of the studies was not performed, since the aim of this integrative review was to provide an overview and synthesis of the existing knowledge from a broader perspective (The Joanna Briggs Institute, 2015).

\section{Results}

\section{Theoretical analysis of the nursing diagnosis DTPs}

The nursing diagnosis DTPs has been analyzed in different NANDA-I conferences. In the 1995 conference, the working group led by Bondy (1995) concluded that the diagnosis DTPs referred to a general and abstract phenomenon and could be articulated in four subcategories: distorted thought, impaired cognition, altered level of consciousness and acute confusion.

In the NANDA-I conference celebrated in 1999, Harvey and colleagues proposed a new definition for the diagnosis DTPs: "A state in which a person expresses or reports fixed false individually held beliefs or experiences inconsistent with reality" (Harvey et al., 1999, p. 604). Furthermore, they suggested additional defining characteristics for the diagnosis: delusions, hallucinations, ideas of reference, magical thinking, obsession, thought insertion, distorted memory, egocentricity, loosening of association, thought broadcasting, illusions and bizarre behaviors.

In addition to the work developed within NANDA-I's context, several authors offer different theoretical propositions that complement each one of the elements of DTPs. 
The definition of this diagnosis was assessed as abstract and difficult to understand. Carpenito (2003) completed the definition of the diagnosis clarifying that the disturbed thought processes was "the state in which an individual experiences a disruption in such mental activities as conscious thought, reality orientation, problem-solving, judgement, and comprehension related to coping (personality and mental) disorders" (p. 590). In their handbook, Wilkinson and Ahern (2008) pointed out that the altered cognitive activities in patients with DTPs include conscious thought, reality orientation, problem-solving and judgement.

The defining characteristics of the nursing diagnosis DTPs have been criticized for being not specific enough and for their lack of operability (Berry, 1987; Hancock, Munjas, Berry, \& Jones, 1994; Ugalde Apalategui \& Rigol i Cuadra, 1995). As it can be observed in table 1, some authors suggested additional defining characteristics in an attempt to complete and clarify the list included in the NANDA-I classification (Carpenito, 2003; Doenges \& Moorhouse, 1992; Kim et al., 1994). 
Table 1. Additional defining characteristics proposed by Doenges \& Moorhouse (1992), Kim (1994) and Carpenito (2003)

\begin{tabular}{|c|c|c|c|}
\hline \multirow[b]{2}{*}{ Defining characteristics } & \multicolumn{3}{|c|}{ Proposed by } \\
\hline & $\begin{array}{c}\text { Doenges \& } \\
\text { Moorhouse, } 1992\end{array}$ & $\begin{array}{l}\text { Kim, } \\
1994\end{array}$ & $\begin{array}{l}\text { Carpenito, } \\
2003\end{array}$ \\
\hline $\begin{array}{l}\text { Imprecise interpretation of internal and/or } \\
\text { external stimuli }\end{array}$ & $X$ & $X$ & $X$ \\
\hline Ideas of reference, hallucinations, fabulation & $X$ & $X$ & $\mathrm{X}$ \\
\hline $\begin{array}{l}\text { Inappropriate non-reality based thinking, } \\
\text { delusions }\end{array}$ & $\mathrm{X}$ & $X$ & $X$ \\
\hline $\begin{array}{l}\text { Impaired ability to: decision-making, problem- } \\
\text { solving, reasoning, abstraction or } \\
\text { conceptualization, calculation }\end{array}$ & $X$ & $X$ & $X$ \\
\hline Memory deficit & $\mathrm{X}$ & $X$ & $\mathrm{X}$ \\
\hline Disorientation in space, time or person & $\mathrm{X}$ & $\mathrm{X}$ & $\mathrm{X}$ \\
\hline Cognitive dissonance & $\mathrm{X}$ & $\mathrm{X}$ & \\
\hline $\begin{array}{l}\text { Hypervigilancie/hypovigilancie } \\
\text { (lightheadedness, confussion) }\end{array}$ & $X$ & $X$ & $X$ \\
\hline Distractibility, impaired attention span & $\mathrm{X}$ & $\mathrm{X}$ & \\
\hline Egocentricity & $\mathrm{X}$ & $\mathrm{X}$ & \\
\hline Obssesions & & $\mathrm{X}$ & $\mathrm{X}$ \\
\hline Inappropriate social behaviour & $X$ & $\mathrm{X}$ & $\mathrm{X}$ \\
\hline Impaired ability to follow comands & & $\mathrm{X}$ & \\
\hline Disturbed sleep pattern & & $\mathrm{X}$ & \\
\hline Inappropiriate affect & & $\mathrm{X}$ & \\
\hline Suspicion & & & $\mathrm{X}$ \\
\hline Phobias & & & $X$ \\
\hline Ritual behaviour & & & $\mathrm{X}$ \\
\hline Impulsiveness & & & $\mathrm{X}$ \\
\hline
\end{tabular}

The last version of the nursing diagnosis DTPs presented in the NANDA-I classification did not include related factors as these were awaiting development (NANDA International, 2008). However, several authors suggested some possible related factors (see table 2) (Carpenito, 2003; Doenges \& Moorhouse, 1992; Garrido Abejar, 2000). Additionally, Wilkison and Ahern (2008) added substance abuse as a potential related factor. 
Table 2. Related factors proposed by Carpenito (2003), Doenges \& Moorhouse (1992) and Garrido Abejar (2000)

\begin{tabular}{|c|c|c|}
\hline \multicolumn{3}{|c|}{ Related factors proposed by } \\
\hline $\begin{array}{l}\text { Carpenito } \\
(\mathbf{2 0 0 3})\end{array}$ & $\begin{array}{l}\text { Doenges \& Moorhouse } \\
(1992)\end{array}$ & $\begin{array}{c}\text { Garrido Abejar } \\
(2000)\end{array}$ \\
\hline \multicolumn{3}{|l|}{ Physiopathological } \\
\hline Degenerative brain injury & \multirow{3}{*}{$\begin{array}{l}\text { Brain injuries } \\
\text { Hypoxia }\end{array}$} & \multirow{2}{*}{$\begin{array}{l}\text { Neurodegenerative } \\
\text { disorders }\end{array}$} \\
\hline Acute brain injury & & \\
\hline $\begin{array}{l}\text { Physiological alterations, due to } \\
\text { withdrawal from alcohol or drugs }\end{array}$ & & $\begin{array}{l}\text { Substance use disorder or } \\
\text { withdrawal syndrome }\end{array}$ \\
\hline \multicolumn{3}{|l|}{ Biochemical disorders } \\
\hline & & Psychiatric disorders \\
\hline \multicolumn{3}{|l|}{ Situational } \\
\hline Continuing hypostimulation & & Social isolation \\
\hline $\begin{array}{l}\text { Abuse (physical, sexual, mental) } \\
\text { Torture }\end{array}$ & & $\begin{array}{l}\text { Antecedents of sexual or } \\
\text { physical abuse }\end{array}$ \\
\hline Emotional trauma & \multirow{5}{*}{ Psychological conflicts } & Emotional trauma \\
\hline Childhood trauma & & \\
\hline Intense anxiety & & \\
\hline Represssed fears & & \\
\hline $\begin{array}{l}\text { Impaired attention span and ability to } \\
\text { reason due to depression, fear, anxiety } \\
\text { or grieving }\end{array}$ & & Severe depression \\
\hline \multicolumn{3}{|l|}{ Maturing } \\
\hline Elderly; isolation, late-life depression & Aging & \\
\hline & Sleep deprivation & \\
\hline
\end{tabular}

Another significant theoretical reference to the diagnosis DTPs is the proposal developed by Hall (1988) about the nursing care of patients with DTPs due to progressive organic degeneration of the cerebral cortex. This author argued that although this etiological factor does not have a potential solution, nursing professionals may act in order to minimize the manifestations associated with it. With this purpose, he proposed the progressively lowered stress threshold model (Hall \& Buckwalter, 1987), that suggests that patients with dementia lose their ability to manage and cope with stress, which causes behavior alterations. In order to prevent or reduce these, the model proposes that nursing care should be focused on minimizing stressor factors such as fatigue; change of environment, caregiver or routine; misleading, overwhelming, or competing stimuli; demands which exceed functional capacity and physical stressors (Hall, 1988). This model has been implemented at numerous healthcare settings, showing positive results (Smith, Gerdner, Hall, \& Buckwalter, 2004). 
Although NANDA-I classification is widely applied, there exist other nursing terminologies. The classification of nursing diagnoses developed by Campbell (1994) contains the diagnosis "illogical thought process", defined as unreasonable or defective thinking. As etiological factors, Campbell suggested some mental disorders (i.e. major depression, psychosis or schizophrenia) and different psychosocial factors such as the lack of ability to resolve problems or to learn alternative behaviors. As defining characteristics, this author proposed the disorganization of ideas and the lack of connection and continuity in thinking (Campbell, 1994).

\section{Empirical data about the nursing diagnosis DTP}

Among the empirical evidence found in relation to the nursing diagnosis DTPs, the majority of papers offer data on the frequency of the diagnosis in different clinical settings. Furthermore, four studies present additional information about the DTPs.

\section{Prevalence studies}

Table 3 illustrates the studies that offer data about the frequency of DTPs. The selected papers were classified into four categories (i.e. specific patient population: psychiatric patients; specific patient population: geriatric patients; specific healthcare setting: community settings; specific healthcare setting: hospitalization). 
Table 3. Prevalence studies providing data about DTPs

\begin{tabular}{|c|c|c|c|}
\hline $\begin{array}{l}\text { REFERENCE } \\
\text { COUNTRY }\end{array}$ & $\begin{array}{c}\text { DESIGN } \\
\text { METHODOLOGY } \\
\end{array}$ & $\begin{array}{l}\text { SETTING } \\
\text { SAMPLE } \\
\end{array}$ & FREQUENCY OF DTPs \\
\hline \multicolumn{4}{|c|}{ SPECIFIC PATIENT POPULATION: PSYCHIATRIC PATIENTS } \\
\hline $\begin{array}{l}\text { Escalada-Hernández et al, } 2015 \\
\text { Escalada-Hernández \& Marín- } \\
\text { Fernández, } 2016 \\
\text { Spain }\end{array}$ & $\begin{array}{l}\text { Multicentric, retrospective, descriptive } \\
\text { ND Identification: Clinical records review }\end{array}$ & $\begin{array}{l}5 \text { Psychiatric clinics (Medium- and } \\
\text { long-term units) } \\
\mathrm{N}=624 \text { psychiatric and psychogeriatric } \\
\text { patients }\end{array}$ & $\begin{array}{l}39.4 \%(\mathrm{n}=246) \\
\text { DTPs was the second most frequent } N D\end{array}$ \\
\hline $\begin{array}{l}\text { Ugalde Apalategui et al., } 2011 \\
\text { Spain }\end{array}$ & $\begin{array}{l}\text { Multicentric, cross-sectional, descriptive } \\
\text { ND Identification: Clinical records review }\end{array}$ & $\begin{array}{l}5 \text { Acute psychiatric units } \\
\mathrm{N}=907 \text { psychiatric patients }\end{array}$ & $\begin{array}{l}45.7 \%(\mathrm{n}=360) \\
\text { DTPs was the second most frequent } N D\end{array}$ \\
\hline $\begin{array}{l}\text { Lluch Canut et al., } 2009 \\
\text { Spain }\end{array}$ & $\begin{array}{l}\text { Descriptive, retrospective } \\
\text { ND Identification: Direct observation of patients }\end{array}$ & $\begin{array}{l}\text { Community-based mental health care } \\
\mathrm{N}=66 \text { patients with serious mental } \\
\text { disorders }\end{array}$ & $\begin{array}{l}54.5 \%(\mathrm{n}=36) \\
\text { DTPs was the third most frequent ND }\end{array}$ \\
\hline $\begin{array}{l}\text { Abderhalden et al., } 2007 \\
\text { Switzerland and Austria }\end{array}$ & $\begin{array}{l}\text { Multicentric, retrospective, descriptive } \\
\text { ND Identification: Clinical records review }\end{array}$ & $\begin{array}{l}11 \text { Acute psychiatric units } \\
\mathrm{N}=330 \text { psychiatric patients }\end{array}$ & $\begin{array}{l}\text { DTPs was the second most frequent ND (information } \\
\text { gathered from summary, frequency was not specified) }\end{array}$ \\
\hline $\begin{array}{l}\text { Sales Orts et al., } 2007 \\
\text { Spain }\end{array}$ & $\begin{array}{l}\text { Analytical, observational, cross-sectional } \\
\text { ND Identification: Nursing records review, verbal } \\
\text { handover information and direct observation }\end{array}$ & $\begin{array}{l}1 \text { Acute psychiatric unit } \\
\mathrm{N}=301 \text { psychiatric patients }\end{array}$ & $\begin{array}{l}94.02 \%(\mathrm{n}=83) \\
\text { DTPs was the most frequent ND }\end{array}$ \\
\hline $\begin{array}{l}\text { Sevillano Arroyo et al., } 2004 \\
\text { Spain }\end{array}$ & $\begin{array}{l}\text { Descriptive, cross-sectional } \\
\text { ND Identification: Clinical records review }\end{array}$ & $\begin{array}{l}1 \text { Acute psychiatric unit } \\
\mathrm{N}=150 \text { psychiatric patients }\end{array}$ & $\begin{array}{l}46 \%(\mathrm{n}=69) \\
\text { DTPs was the third most frequent } N D\end{array}$ \\
\hline $\begin{array}{l}\text { Stefan et al., } 2003 \\
\text { Switzerland and Austria }\end{array}$ & $\begin{array}{l}\text { Descriptive, retrospective } \\
\text { ND Identification: Nursing records review }\end{array}$ & $\begin{array}{l}1 \text { Acute psychiatric unit } \\
\mathrm{N}=30 \text { psychiatric patients }\end{array}$ & $\begin{array}{l}\text { DTPs among the } 3 \text { most frequent ND (information } \\
\text { gathered from summary, frequency was not specified) }\end{array}$ \\
\hline $\begin{array}{l}\text { Boomsma et al., } 1997 \\
\text { Netherlands }\end{array}$ & $\begin{array}{l}\text { Action research } \\
\text { ND Identification: Clinical records review (Content } \\
\text { analysis and coding as ND) }\end{array}$ & $\begin{array}{l}4 \text { crisis-orientated home care teams } \\
\mathrm{N}=61 \text { psychiatric patients }\end{array}$ & $\begin{array}{l}\text { DTPs was the fifth most frequent ND } \\
\text { (frequency not specified) }\end{array}$ \\
\hline $\begin{array}{l}\text { Murphy, } 1992 \\
\text { USA }\end{array}$ & $\begin{array}{l}\text { Descriptive, longitudinal } \\
\text { ND Identification: Analysis of interview transcription }\end{array}$ & $\begin{array}{l}\text { Recruitment from a study of symptom } \\
\text { monitoring after alcohol dependence } \\
\mathrm{N}=26 \text { patients with } 1 \text { year abstinence }\end{array}$ & $16.9 \%(\mathrm{n}=7)$ \\
\hline \multicolumn{4}{|c|}{ SPECIFIC PATIENT POPULATION: GERIATRIC PATIENTS } \\
\hline $\begin{array}{l}\text { Kuzu et al., } 2005 \\
\text { Turkey }\end{array}$ & $\begin{array}{l}\text { Pre-post interventional study } \\
\text { ND Identification: Observación directa del paciente }\end{array}$ & $\begin{array}{l}\text { Community care } \\
\mathrm{N}=32 \text { patients with Alzheimer }\end{array}$ & $\begin{array}{l}\text { Pretest: } 46.8 \%(n=15) \\
\text { Posttest: } 31.3 \%(n=10)\end{array}$ \\
\hline $\begin{array}{l}\text { Barriguete et al., } 2005 \\
\text { Spain }\end{array}$ & $\begin{array}{l}\text { Descriptive, cross-sectional } \\
\text { ND Identification: Direct observation of patients }\end{array}$ & $\begin{array}{l}\text { Nursing home } \\
\mathrm{N}=52 \text { elderly patients }\end{array}$ & $1.9 \%(\mathrm{n}=1)$ \\
\hline
\end{tabular}




\begin{tabular}{|c|c|c|c|}
\hline $\begin{array}{l}\text { REFERENCE } \\
\text { COUNTRY }\end{array}$ & $\begin{array}{c}\text { DESIGN } \\
\text { METHODOLOGY }\end{array}$ & $\begin{array}{l}\text { SETTING } \\
\text { SAMPLE }\end{array}$ & FREQUENCY OF DTPs \\
\hline $\begin{array}{l}\text { Borges Teixeira et al., } 2003 \\
\text { Brazil }\end{array}$ & $\begin{array}{l}\text { Descriptive, exploratory } \\
\text { ND Identification: Direct observation of patients }\end{array}$ & $\begin{array}{l}\text { Open psychiatric clinic } \\
\mathrm{N}=48 \text { elderly patients with cognitive } \\
\text { deterioration }\end{array}$ & $27 \%(n=13)$ \\
\hline $\begin{array}{l}\text { Park et al., } 2004 \\
\text { USA }\end{array}$ & $\begin{array}{l}\text { Descriptive, retrospective } \\
\text { ND Identification: Clinical records review }\end{array}$ & $\begin{array}{l}\text { Community hospital } \\
\mathrm{N}=597 \text { elderly patients with dementia }\end{array}$ & $29 \%(n=144)$ \\
\hline $\begin{array}{l}\text { Daly et al., } 1995 \\
\text { USA }\end{array}$ & $\begin{array}{l}\text { Descriptive, retrospective } \\
\text { ND Identification: Clinical records review }\end{array}$ & $\begin{array}{l}\text { Long-term care institution } \\
\mathrm{N}=29 \text { elderly patients }\end{array}$ & $65.52 \%(\mathrm{n}=19)$ \\
\hline $\begin{array}{l}\text { Mass \& Buckwalter } 1988 \\
\text { USA }\end{array}$ & $\begin{array}{l}\text { Descriptive, cross-sectional } \\
\text { ND Identification: interviews, clinical records review and } \\
\text { questionnaires }\end{array}$ & $\begin{array}{l}\text { Long-term care institution } \\
\mathrm{N}=60 \text { patients with Alzheimer }\end{array}$ & $52 \%(\mathrm{n}=31)$ \\
\hline $\begin{array}{l}\text { Hardy et al., } 1988 \\
\text { USA }\end{array}$ & $\begin{array}{l}\text { Review of } 5 \text { studies } \\
\text { ND Identification: Clinical records review }\end{array}$ & $\begin{array}{l}\text { Nursing homes } \\
\text { Elderly patients included in each study: } \\
\text { - IVH, } 1985(\mathrm{~N}=121) \\
\text { - Hallal } 1985(\mathrm{~N}=106) \\
\text { - Leslie } 1981(\mathrm{~N}=210) \\
\text { - Rantz 1984: }(\mathrm{N}=328) \\
\text { - Rantz 1986 }(\mathrm{N}=328) \\
\end{array}$ & $\begin{array}{l}\text { DTPs found in the } 5 \text { studies (frequency not specified): } \\
\text { - IVH 1985: the third most frequent } N D \\
\text { - Hallal 1985: the eleventh most frequent } N D \\
\text { - Leslie 1981: the third most frequent } N D \\
\text { - Rantz 1984: the third most frequent } N D \\
\text { - Rantz 1986: the third most frequent } N D\end{array}$ \\
\hline \multicolumn{4}{|c|}{ SPECIFIC HEALTHCARE SETTINGS: COMMUNITY SETTING } \\
\hline $\begin{array}{l}\text { Nieto et al., } 2004 \\
\text { Spain }\end{array}$ & $\begin{array}{l}\text { Descriptive, cross-sectional } \\
\text { ND Identification: Direct observation of patients }\end{array}$ & $\begin{array}{l}\text { Home-care service } \\
\mathrm{N}=680 \text { patients }\end{array}$ & $0.92 \%(n=49)$ \\
\hline $\begin{array}{l}\text { Keenan et al., } 2003 \\
\text { USA }\end{array}$ & $\begin{array}{l}\text { Descriptive, cross-sectional } \\
\text { ND Identification: Direct observation of patients }\end{array}$ & $\begin{array}{l}\text { Adult care nurse practitioner setting } \\
\mathrm{N}=319 \text { patients }\end{array}$ & $2.2 \%(\mathrm{n}=3)$ \\
\hline $\begin{array}{l}\text { Clavería Señís, } 1997 \\
\text { Spain }\end{array}$ & $\begin{array}{l}\text { Descriptive, cross-sectional } \\
\text { ND Identification: Clinical records review (content } \\
\text { analysis) }\end{array}$ & $\begin{array}{l}\text { Home-care service } \\
\mathrm{N}=138 \text { patients }\end{array}$ & $\begin{array}{l}11.6 \%(\mathrm{n}=37) \\
\text { DTPs was the eighth most frequent ND }\end{array}$ \\
\hline $\begin{array}{l}\text { Kang et al., } 1994 \\
\text { USA }\end{array}$ & $\begin{array}{l}\text { Descriptive, cross-sectional } \\
\text { ND Identification: Nursing records review }\end{array}$ & $\begin{array}{l}\text { Rural setting of advanced practice } \\
\text { nursing with families with disabled } \\
\text { young children } \\
\mathrm{N}=75 \text { disabled children }\end{array}$ & $\begin{array}{l}\text { DTPs was among the } 5 \text { most frequent ND (frequency not } \\
\text { specified) }\end{array}$ \\
\hline \multicolumn{4}{|c|}{ SPECIFIC HEALTHCARE SETTINGS: HOSPITALIZATION } \\
\hline $\begin{array}{l}\text { Paans \& Müller-Staub, } 2015 \\
\text { Germany }\end{array}$ & $\begin{array}{l}\text { Cross-sectional, descriptive } \\
\text { ND Identification: review of nursing records }\end{array}$ & $\begin{array}{l}10 \text { hospitals } \\
\mathrm{N}=369 \text { hospitalized patients }\end{array}$ & $1.1 \%(n=4)$ \\
\hline
\end{tabular}




\begin{tabular}{|c|c|c|c|}
\hline $\begin{array}{l}\text { REFERENCE } \\
\text { COUNTRY }\end{array}$ & $\begin{array}{c}\text { DESIGN } \\
\text { METHODOLOGY }\end{array}$ & $\begin{array}{l}\text { SETTING } \\
\text { SAMPLE }\end{array}$ & FREQUENCY OF DTPS \\
\hline Soares Novaes et al., 2015 & Descriptive, exploratory and cross-sectional & Surgical clinic & \multirow{2}{*}{$3.5 \%(\mathrm{n}=1)$} \\
\hline Brazil & ND Identification: Direct observation of patients & $\mathrm{N}=28$ surgical patients & \\
\hline \multirow[t]{2}{*}{$\begin{array}{l}\text { Thoroddsen \& Ehnfors, } 2007 \\
\text { Iceland }\end{array}$} & $\begin{array}{l}\text { Pre-post test inverentional study (educational intervention } \\
\text { about nursing documentation) }\end{array}$ & \multirow{2}{*}{$\begin{array}{l}\text { Acute care hospital } \\
\text { Pretest: } N=362 \text { hospitalized patients } \\
\text { Posttest: N=355 hospitalized patients }\end{array}$} & $\begin{array}{l}\text { Pretest: DTPs was the ninth most frequent } N D(\mathrm{n}=37 \text {, } \\
2.4 \%)\end{array}$ \\
\hline & ND Identification: Nursing records review & & $\begin{array}{l}\text { Posttest DTPs was not among the } 10 \text { most frequent ND } \\
\text { (frequency not specified) }\end{array}$ \\
\hline \multirow{2}{*}{$\begin{array}{l}\text { Thoroddsen \& Thorsteinsson, } \\
2002 \\
\text { Iceland }\end{array}$} & Descriptive, retrospective & \multirow{2}{*}{$\begin{array}{l}\text { Acute care hospital } \\
\mathrm{N}=1103 \text { hospitalized patients }\end{array}$} & $2.3 \%(\mathrm{n}=51)$ \\
\hline & $\begin{array}{l}\text { ND Identification: Clinical records review (content } \\
\text { analysis) }\end{array}$ & & $\begin{array}{l}\text { DTPs was the eleventh most frequent ND (frequency not } \\
\text { specified) }\end{array}$ \\
\hline Karpiuk et al., 1997 & Descriptive, retrospective & 8 hospitals & $22.3 \%(\mathrm{n}=42)$ \\
\hline USA & ND Identification: Clinical records review & $\mathrm{N}=188$ patients with fractured femur & $\begin{array}{l}\text { DTPs was the fourth most frequent ND and one of the } \\
\text { most common unresolved ND }\end{array}$ \\
\hline
\end{tabular}

$N D=$ Nursing diagnoses 
The category specific patient population: psychiatric patients includes six studies developed in psychiatric inpatient care facilities (Abderhalden et al., 2007; EscaladaHernández \& Marín-Fernández, 2016; Sales Orts, 2005; Stefan et al., 2003; Ugalde Apalategui \& Lluch Canut, 2011) and two in community psychiatric care services (Boomsma, Dingemans, \& Dassen, 1997; Lluch Canut et al., 2009). Additionally, another study on symptom monitoring post alcohol dependence show data about the frequency of DTPs (Murphy, 1992). As it can be observed in table 3, almost all studies concluded that the diagnosis DTPs was among the five most common diagnoses, with a frequency between $40 \%$ and $50 \%$ in the majority of them.

Furthermore, two of the papers within this category (Escalada-Hernández et al., 2015; Ugalde Apalategui \& Lluch Canut, 2011) offer information about patients' psychiatric diagnosis. Thus, both of them suggested that the frequency of DTPs was higher than $30 \%$ in patients with medical diagnoses such as schizophrenia, disorders of adult personality and behaviors, substance use disorders, depression or bipolar disorders. On the other hand, one of these studies found a statistically significant relationship between the presence of DTPs and, both, a higher degree of severity of problems associated with mental illness and a higher number of nursing diagnoses recorded in the care plan (Escalada-Hernández \& MarínFernández, 2016).

Seven studies including geriatric patients were found (table 3). In these studies, a high variability in the frequency of DTPs is observed. This can be explained because of the existing differences between them in relation to aspects such as clinical setting and patient profile. As it can be noted, in institutionalized elderly patients with dementia and cognitive deterioration the nursing diagnosis DTPs was very frequent (52\%) (Maas \& Buckwalter, 1988). In the case of elderly with dementia in the community setting, the frequency of this 
diagnosis was lower, being between $31.3 \%$ and 25\% (Borges Teixeira \& Quintella Fernandes, 2003; Kuzu et al., 2005). Park and colleagues (2004) identified the diagnosis DTPs in $29 \%$ of their sample of hospitalized elderly patients with dementia. Some of the studies on institutionalized patients do not specify the level of cognitive impairment and thus, show discordant results about the frequency of DTPs (Barrigüete Andreu \& González Porras, 2005; Daly, Maas, \& Buckwalter, 1995; Hardy, Maas, \& Akins, 1988).

As it could be expected, the research projects developed in the community setting (excluding those with elderly population already presented) found a lower frequency of the diagnosis DTPs, between 0.9\% and 11\% (Clavería Señis, 1997; Keenan, Stocker, Barkauskas, Treder, \& Heath, 2003; Nieto García et al., 2004). This category also includes Kang and colleagues' study (1994) implemented in a rural setting of advanced practice nursing with families with disabled young children. Its findings suggested that DTPs was among the five most frequent nursing diagnoses among children. This is the only study that was found with pediatric population.

The frequency of the nursing diagnosis DTPs in hospitalization units is very low, being between $1 \%$ and $3.5 \%$ in four of the studies in this category (Paans \& MüllerStaub, 2015; Soares Novaes, Morbin Torres, \& Vilcinski Oliva, 2015; Thoroddsen \& Ehnfors, 2007; Thoroddsen \& Thorsteinsson, 2002). As it can be observed in table 3, the exception in this group is the research led by Karpiuk (Karpiuk, Delaney, \& Ryan, 1997), in which the frequency of DTPs was $22.3 \%$ among patients who had a fractured femur. This finding is consistent with the fact that this type of patients may have a higher risk of suffering from delirium.

In addition to studies presented in table 3, a qualitative study illustrating the presence of the diagnosis DTPs was found (Carlson-Catalano, 1998). In this research, nine post-acute-phase battered women who lived in rural areas were interviewed using Gordon's 
(1996) functional health patterns as a framework. All of them presented the nursing diagnosis DTPs.

\section{Other studies}

In the literature search, four additional studies presenting additional data on the nursing diagnosis DTPs were found. Hancock and colleagues (1994), applying the diagnostic content validation model (Fehring, 1987), asked to 66 members of NANDA-I to assess the defining characteristics of the nursing diagnoses DTPs and sensory/perceptual alterations (00122). In the case of DTPs, two defining characteristics were identified as major: impaired ability to problem solve and impaired ability to make decisions. Additionally, participants were required to identify the most representative characteristics for each particular diagnosis. For DTPs, these were disordered thought sequencing, nonreality-based thinking, impaired ability to problem solve and altered abstraction. Regarding their use in clinical practice, $86 \%$ of respondents considered that both nursing diagnoses were within the domain of nursing practice (Hancock et al., 1994).

A more recent study developed in Taiwan (Chung, Chiang, Chou, Chu, \& Chang, 2010) explored the intra-rater and inter-rater reliability of the nursing process records in psychiatry. Nursing diagnoses were identified based on the signs and symptoms described in the assessment interviews for 54 patients with schizophrenia. The most frequent used diagnosis was DTPs, showing a moderate level of inter-rater reliability (kappa=0.53, 95\% CI: 0.26-0.80) and a high level of intra-rater reliability (kappa=0.77, 95\% CI: 0.56-0.98). In the analysis of each defining characteristic of DTPs, adequate levels of intra-rater reliability were found in all of them (range: $0.30-0.79$ ).

In the Spanish context, a validation study of the most common nursing diagnoses in four clinical areas, including Mental Health, was implemented in the mid-90's (González Carrión et al., 1994, 1997). In the first phase of this research, the most frequently used nursing 
diagnoses in each area were identified. In the second phase, a concordance study was developed. Two nurses directly assessed 30 patients in each area, in order to evaluate whether they had the nursing diagnoses described in the first phase. The nursing diagnosis DTPs was labelled exclusively in mental health, where it was identified in 23 of the 30 patients, showing an excellent kappa index $(0,930)$.

Finally, another relevant paper is the literature review developed by Frauenfelder and colleagues (2011) aimed at identifying the documented patients' responses to actual or potential health problems/life processes addressed by nurses in the field of mental health. They identified 21 phenomena recognizable as NANDA-I nursing diagnoses and 43 phenomena that were not included in this classification. Among those, there was the phenomenon disturbed thinking patterns that comprised terms such as difficulty in expressing a complete thought or opinion, excessive thinking or disorganized thought processes. According to these authors, this phenomenon could be consistent with DTPs and they encouraged the debate about its reintroduction in the NANDA-I classification.

\section{Discussion}

The aim of this review was to synthetize the existing data on the nursing diagnosis DTPs, integrating empirical evidence and theoretical reports. From the theoretical point of view, the revised papers highlight that the diagnosis DTPs could be excessively abstract, making difficult its use in the clinical practice. Since 80 's, several authors have proposed alternative definitions and additional defining characteristics and related factors with the purpose of clarifying and complementing this nursing diagnosis. However, these suggestions have not achieved that DTPs could be considered a more precise and unambiguous nursing diagnosis.

It could be argued that the main reason for the lack of clarity in relation to the nursing diagnosis DTPs is the complexity of the constructs thought and cognition. In the field of the 
cognitive psychology, Ortiz Ocaña (2009) explains that the cognition involves different mental processes that take place between the perception of the stimuli and the response to it. This author categorizes as complex cognitive processes the thought, language, intelligence and creativity; and as simple cognitive processes the sensation, perception, attention, concentration, memory and imagination. Assessing the proposed definitions and defining characteristics for the nursing diagnosis DTPs using this classification as a framework, it can be observed that they do not exclusively focus on the cognitive process of thought, mixing and referring to several cognitive processes. This highlights the lack of conceptual clarity relating to this diagnosis and emphasizes the need of a further development of its conceptual basis.

In relation to empirical evidence, most of the research papers found offer data about the frequency of DTPs in different clinical settings. This nursing diagnosis is very common in patients with mental health problems, either with psychiatric disorders or with cognitive impairments or dementia. Because of this, DTPs may be very prevalent in clinical settings in the field of mental health or geriatrics.

Although a formal assessment of the quality was not undertaken, it is worth mentioning some methodological aspects about the research papers found. One important point is how the frequency of nursing diagnoses was analyzed in those studies. Many of the studies presented in table 3 examined retrospectively the frequency of the nursing diagnoses documented in clinical records. This kind of design is very common because of its ease of implementation, being one of the advantages and applications of the integration of the NANDA-I classification in electronic clinical records (NANDA International, 2008). However, this type of studies show information about the documented problems, and not about the direct observation of patients' state. In the case of DTPs, it could be argued that its identification might be difficult and therefore, its presence may not be detected and registered 
adequately. Roberts and colleagues (1996), who compared the documented nursing diagnoses to supporting clinical evidence obtained from subjects, demonstrated this difficulty. Their global results revealed a very low congruence level, being DTPs one of the less documented nursing diagnoses.

Hancock and colleagues' research (1994) is the only validation study for the nursing diagnosis DTPs developed to date. One of the critical aspects of this type of studies is the selection of the "true experts" as the validity of the results is based on their opinion (Fehring, 1994). However, the selection criteria applied in this study was not described.

An interesting finding of this review is that none of the proposals related to the nursing diagnosis DTPs made by different authors since the 80's was incorporated into the version included in the NANDA-I classification. As it has been illustrated, although the relevant works are limited in number and scope, some of their results could have enriched the existing version of the diagnosis DTPs.

The main conclusion that could be drawn from this review is that the existing version of the nursing diagnosis DTPs was not satisfactorily developed from a conceptual point of view and that the available empirical data on this nursing diagnosis is very limited. This justified its exclusion from the taxonomy (Herdman, 2012). Therefore, it could be argued that the development of a new version of this nursing diagnosis is essential. The process of development of this new proposal should begin by establishing its theoretical and conceptual basis (Fehring, 1994; Whitley, 1999). The first step in this process should be the identification and formulation of the focus of the diagnosis, that is, a concept which represents a specific phenomenon of interest for the nursing discipline (Avant, 1990). In order to do that, Walker \& Avant (2011) recommend implementing a conceptual work following systematic methods. 


\section{Conclusion}

The literature synthetized demonstrates that there exists an interest in the nursing diagnosis DTPs. However, the available evidence is not very extensive and further work, at conceptual and empirical level, is necessary in order to complete and refine this nursing diagnosis.

\section{Implications for nursing}

The reinclusion of the nursing diagnosis DTPs in the NANDA-I classification will specially contribute to increment its utility in mental health care, by incorporating a phenomenon frequently addressed in this context, thus, supporting the rigorous documentation of nursing care.

However, in order to achieve its reinclusion further research on this nursing diagnosis is necessary, including the clarification of its conceptual base, the establishment of its content validation and the implementation of multiple studies of this nursing phenomena in the clinical setting.

\section{Acknowledgements}

This study was part of the PhD dissertation of the first author, and supervised by the second author.

The authors would like to thank Sr Nieves Escalada ODN and Ms Jenny Smith for their valuable review of the manuscript for English usage.

\section{References}

Abderhalden, C., Needham, I., Faust, A. M., Grywa, D., Quiblier, U., Stefan, H., \& Willard, W. (2007). [Nursing diagnoses in acute psychiatric wards in Switzerland and Austria: commonalities and differences]. Pflege, 20(3), 149-156.

Ackley, B. J., \& Ladwig, G. B. (2007). Manual de diagnósticos de enfermería. Guía para la planificación de los cuidados. ( $7^{\mathrm{a}} \mathrm{ed}$.). Barcelona: Elsevier Mosby.

Avant, K. C. (1990). The art and science in nursing diagnosis development. International Journal of Nursing Terminologies and Classifications, 1(2), 51-56. https://doi.org/http://dx.doi.org/10.1111/j.1744-618X.1990.tb00236.x

Barrigüete Andreu, M. I., \& González Porras, C. (2005). Prevalencia de los diagnósticos enfermeros 
en una residencia geriátrica. Hygia, 72, 50-54.

Berry, K. N. (1987). Let's create diagnoses psych nurses can use. AJN The American Journal of Nursing, 87(5), 707-708.

Bondy, K., Maas, M., McCourt, A., \& Rantz, M. J. (1995). Altered thought process. In M. J. Rantz \& P. LeMone (Eds.), Classification of nursing diagnoses: proceedings of the eleventh conference, North American Nursing Diagnosis Association. (pp. 401-404). Glendale, CA: CINAHL Information Systems.

Boomsma, J., Dingemans, C. A., \& Dassen, T. W. (1997). The nursing process in crisis-oriented psychiatric home care. Journal of Psychiatric and Mental Health Nursing, 4(4), 295-301.

Borges Teixeira, M., \& Quintella Fernandes, R. A. (2003). Diagnósticos de enfermagem identificados em idosos com distúrbio mental. Revista Brasileira de Enfermagem, 56(6), 619-623. https://doi.org/10.1590/S0034-71672003000600005

Campbell, C. (1994). Enfermería: Diagnósticos y Métodos. Granollers: Marín D.L.

Carlson-Catalano, J. (1998). Nursing diagnoses and interventions for post-acute-phase battered women. International Journal of Nursing Terminologies and Classifications, 9(3), 101-110. https://doi.org/10.1111/j.1744-618X.1998.tb00154.x

Carpenito, L. J. (2003). Diagnósticos de enfermería. Aplicaciones a la práctica clínica. (9th ed.). Madrid: McGraw-Hill Interamericana.

Chung, M. H., Chiang, I. J., Chou, K. R., Chu, H., \& Chang, H. J. (2010). Inter-rater and intra-rater reliability of nursing process records for patients with schizophrenia. Journal of Clinical Nursing, 19(21-22), 3023-3030. Retrieved from http://dx.doi.org/10.1111/j.13652702.2010.03301.x

Clavería Señis, M. (1997). Diagnósticos de enfermería utilizados por un equipo de atención domiciliaria socio-sanitaria. Enfermería Científica, 182-183, 49-54.

Daly, J. M., Maas, M., \& Buckwalter, K. (1995). Use of standardized nursing diagnoses and interventions in long-term care. Journal of Gerontological Nursing, 21(8), 29-36.

Doenges, M. E., \& Moorhouse, M. F. (1992). Guía de bolsillo de diagnósticos y actuaciones de enfermería. Barcelona: Doyma Enfermería.

Echevarría Pérez, P., Romero Sánchez, J. M., Giró Formatger, D., \& Giménez Fernández, M. (Eds.). (2016). Investigación en metodología y lenguajes enfermeros. Barcelona: Elsevier, AENTDE.

Escalada-Hernández, P., \& Marín-Fernández, B. (2016). The nursing diagnosis disturbed thought processes in psychiatric patients: prevalence and associated characteristics. International Journal of Nursing Knowledge, 27(3), 156-161. https://doi.org/10.1111/2047-3095.12091

Escalada-Hernández, P., Muñoz-Hermoso, P., González-Fraile, E., Santos, B., González-Vargas, J. A., Feria-Raposo, I., ... González, E. N. (2015). A retrospective study of nursing diagnoses, outcomes, and interventions for patients with mental disorders. Applied Nursing Research, 28(2), 92-98. https://doi.org/doi.org/10.1016/j.apnr.2014.05.006

Escalada Hernández, P., Muñoz Hermoso, P., \& Marro Larrañaga, I. (2013). Atención de enfermería a pacientes psiquiátricos NANDA-NIC-NOC: una revisión de la literatura [Nursing care for psychiatric patients defined by NANDA-NIC-NOC terminology: a literature review]. Revista ROL de Enfermería, 36(3), 14-6, 19-25.

Fehring, R. J. (1987). Methods of validate nursing diagnoses. Hearth \& Lung, 16(6), 625-629.

Fehring, R. J. (1994). The Fehring model. In Classification of nursing diagnoses: proceeding of the Tenth Conference of North American Nursing Diagnosis Association. (pp. 55-62). Philadelphia USA: JB Linppincottp.

Frauenfelder, F., Müller-Staub, M., Needham, I., \& Van Achterberg, T. (2011). Nursing phenomena in inpatient psychiatry. Journal of Psychiatric and Mental Health Nursing, 18(3), 221-235. https://doi.org/10.1111/j.1365-2850.2010.01659.x

Frauenfelder, F., Van Achterberg, T., Needham, I., \& Müller Staub, M. (2016). Nursing diagnoses in inpatient psychiatry. International Journal of Nursing Knowledge, 27(1), 24-34. https://doi.org/10.1111/2047-3095.12068

Garrido Abejar, M. (2000). Problemas de salud mental desde la perspectiva enfermera. In F. Mejías Lizancos \& M. D. Serrano Parra (Eds.), Enfermería en Psiquiatría y Salud Mental (pp. 319361). Madrid: DAE.

González Carrión, P., Hernández Padilla, M., Rivas Campos, A., Toral López, I., Sánchez Martínez, 
P., Quero Rufián, A., \& Ramírez Sánchez, M. D. (1994). Validación de los diagnósticos de enfermería de la NANDA: una experiencia en cuatro áreas asistenciales. Enfermería Clínica, 4(3), 124-127.

González Carrión, P., Rivas Campos, A., Toral López, I., Hernández Padilla, M., Sánchez Martínez, P., Quero Rufián, A., \& Ramírez Sánchez, M. D. (1997). Validación de las categorías diagnósticas de la NANDA en cuatro áreas asistenciales. Enfermería Clínica, 7(1), 5-12.

Gordon, M. (1996). Diagnósticos de Enfermería: Proceso y Aplicación (3ª). Madrid: Mosby/Doyma Libros.

Gordon, M., \& Sweeney, M. A. (1979). Methodological problems and issues in identifying and standardizing nursing diagnoses. Advances in Nursing Science, 2(1), 1-16.

Hall, G. R. (1988). Focus: Nursing diagnosis: Alterations in thought process. Journal of Gerontological Nursing, 14(3), 30.

Hancock, C. K., Munjas, B., Berry, K., \& Jones, J. (1994). Altered thought processes and Sensory / perceptual alterations: a critique. International Journal of Nursing Terminologies and Classifications, 5(1), 26-30. https://doi.org/10.1111/j.1744-618X.1994.tb00364.x

Hardy, M., Maas, M., \& Akins, J. (1988). The prevalence of nursing diagnoses among elderly and long term care residents: A descriptive comparison. Recent Advances in Nursing, 21, 144-158.

Harvey, R., McComb, P., McLaughlin, D., Moorhouse, M., Rahal, L., Semenza, N., ... Hancock, C. (1999). Altered thought process. In M. J. Rantz (Ed.), Classification of nursing diagnoses: proceedings of the thirteenth conference, North American Nursing Diagnosis Association. Celebrating the 25th anniversary of NANDA. (pp. 604-605). Glendale, CA: Cinahl Information Systems.

Hederman, T. H., \& Kamitsuru, S. (Eds.). (2014). NANDA International nursing diagnoses: definitions and classification, 2015-2017. Oxford: Wiley-Blackwell.

Herdman, T. H. (Ed.). (2012). NANDA International Nursing diagnoses: Definitions and classification 2012-2014. Oxford: Wiley-Blackwell.

Kang, R., Barnard, K., \& Oshio, S. (1994). Description of the clinical practice of advanced practice nurses in family-centered early intervention in two rural settings. Public Health Nursing, 11(6), 376-384. https://doi.org/10.1111/j.1525-1446.1994.tb00202.x

Karpiuk, K. L., Delaney, C. W., \& Ryan, P. (1997). South dakota statewide nursing minimum data set project. Journal of Professional Nursing, 13(2), 76-83. https://doi.org/doi.org/10.1016/S87557223(97) $80007-5$

Keenan, G., Stocker, J., Barkauskas, V., Treder, M., \& Heath, C. (2003). Toward collecting a standardized nursing data set across the continuum: case of adult care nurse practitioner setting. Outcomes Management, 7(3), 113-120.

Kim, M. J., McFarland, G. K., \& McLane, A. M. (1994). Diagnóstico en enfermería: guía clínica de enfermería. Barcelona: Mosby.

Kuzu, N., Beşer, N., Zencir, M., Şahiner, T., Nesrin, E., Ahmet, E., ... Çaĝdaş, E. (2005). Effects of a comprehensive educational program on quality of life and emotional issues of dementia patient caregivers. Geriatric Nursing, 26(6), 378-386. https://doi.org/doi.org/10.1016/j.gerinurse.2005.09.015

Lluch Canut, M. T., Sabadell Gimeno, M., Puig Llobet, M., Cencerrado Muñoz, M. A., Navas Alcal, D., Ferret Canale, M., ... Ugalde Apalategui, M. (2009). Diagnósticos enfermeros en pacientes con trastorno mental severo con seguimiento de enfermería en centros de salud mental. Revista Presencia, 5(10). Retrieved from http://www.index-f.com/presencia/n10/p7151.php

Luis, M. T. (1998). Diagnósticos Enfermeros : un instrumento para la práctica asistencia. Madrid: Harcourt Brace.

Luis Rodrigo, M. T. (2008). Los Diagnósticos Enfermeros : revisión crítica y guía práctica. Barcelona: Elsevier Masson.

Maas, M. L., \& Buckwalter, K. C. (1988). A special Alzheimer's unit: Phase I baseline data. Applied Nursing Research, 1(1), 41.

Murphy, S. A. (1992). Validation of addictions nursing diagnoses in a sample of alcohol abstainers 1 year posttreatment. Archives of Psychiatric Nursing, 6(6), 340-346. https://doi.org/10.1016/0883-9417(92)90086-X

NANDA International. (2008). Nursing Diagnoses: Definitions and Classificacion 2009-2011. 
Oxford: Wiley-Blackwell.

Nieto García, E., Domínguez Ruiz, M., Casado Lollano, A., Monleón Just, M., Díz Gómez, J., Sánchez Pérez, C., ... Domingo Martón, R. (2004). Diagnósticos enfermeros en la atención domiciliaria. Metas de Enfermería, 7(3), 6-12.

Ortiz Ocaña, A. L. (2009). Aprendizaje y comportamiento basados en el funcionamiento del cerebro humano: emociones, procesos cognitivos, pensamientos e inteligencia. Hacia una teoría del aprendizaje neuroconfigurador. Barranquilla (Colombia): Ediciones Litoral.

Paans, W., \& Müller-Staub, M. (2015). Patients' care needs: documentation analysis in general hospitals. International Journal of Nursing Knowledge, 26(4), 178-186. https://doi.org/10.1111/2047-3095.12063

Park, M., Delaney, C., Maas, M., \& Reed, D. (2004). Using a Nursing Minimum Data Set with older patients with dementia in an acute care setting. Journal of Advanced Nursing, 47(3), 329-339. Retrieved from http://dx.doi.org/10.1111/j.1365-2648.2004.03097.x

Pompeo, D. A., Rossi, L. A., \& Galvão, C. M. (2009). Revisão integrativa: etapa inicial do processo de validação de diagnóstico de enfermagem. Acta Paulista de Enfermagem, 22(4), 434-438. https://doi.org/10.1590/S0103-21002009000400014

Sales Orts, R. (2005). Análisis del proceso de cuidados de enfermería en una sala de psiquiatría (Doctoral dissertation). Universidad de Sevilla, Sevilla. Retrieved from http://fondosdigitales.us.es/tesis/tesis/2742/analisis-del-proceso-de-cuidados-de-enfermeria-enuna-sala-de-psiquiatria/

Smith, M., Gerdner, L. A., Hall, G. R., \& Buckwalter, K. C. (2004). History, development, and future of the Progressively Lowered Stress Threshold: a conceptual model for dementia care. Journal of the American Geriatrics Society, 52(10), 1755-1760. https://doi.org/10.1111/j.15325415.2004.52473.x

Soares Novaes, E., Morbin Torres, M., \& Vilcinski Oliva, A. P. (2015). Nursing diagnoses in surgical clinic. Acta Paulista de Enfermagem, 28(1), 26-31. https://doi.org/10.1590/19820194201500006

Stefan, H., Grywa, D., Abderhalden, C., Faust, A. M., Needham, I., Quiblier, U., \& Willard, W. (2003). Nursing diagnoses in psychiatric acute wards in Switzerland and Austria. International Journal of Nursing Terminologies and Classifications, 14(Sup. 4), S22-S23. https://doi.org/10.1111/j.1744-618X.2003.017_9.x

The Joanna Briggs Institute. (2015). The Joanna Briggs Institute Reviewers' Manual 2015: Methodology for JBI scoping reviews. Joanna Briggs Institute, 1-24. https://doi.org/10.1017/CBO9781107415324.004

Thoroddsen, A., \& Ehnfors, M. (2007). Putting policy into practice: pre- and posttests of implementing standardized languages for nursing documentation. Journal of Clinical Nursing, 16(10), 1826-1838. https://doi.org/10.1111/j.1365-2702.2007.01836.x

Thoroddsen, A., \& Thorsteinsson, H. S. (2002). Nursing diagnosis taxonomy across the Atlantic Ocean: congruence between nurses' charting and the NANDA taxonomy. Journal of Advanced Nursing, 37(4), 372-381. https://doi.org/10.1046/j.1365-2648.2002.02101.x

Ugalde Apalategui, M., \& Lluch Canut, M. T. (2011). Estudio multicéntrico del uso y utilidad de las Taxonomías Enfermeras en Unidades de Hospitalización Psiquiátrica. [Informe de trabajo publicado bajo licencia Creative Commons. Diposit Digital de la Universitat de Barcelona, apartat Recerca.]. Retrieved from http://hdl.handle.net/2445/19207

Ugalde Apalategui, M., \& Rigol i Cuadra, A. (1995). Diagnósticos de enfermería, taxonomía NANDA I traducción, revisión y comentarios. Barcelona: Masson.

Walker, L. O., \& Avant, K. C. (2011). Strategies for theory construction in nursing (5º. Madrid: Prentice Hall Pearson.

Whitley, G. G. (1999). Processes and methodologies for research validation of nursing diagnoses. International Journal of Nursing Terminologies and Classifications, 10(1), 5-14. https://doi.org/10.1016/S0883-9417(97)80035-0

Whittemore, R., \& Knafl, K. (2005). The integrative review: Updated methodology. Journal of Advanced Nursing, 52(5), 546-553. https://doi.org/10.1111/j.1365-2648.2005.03621.x

Wilkinson, J. M., \& Ahern, N. R. (2008). Manual de diagnósticos de enfermería (9a ed.). Madrid: Pearson Education. 
Disturbed Thought Processes 